\title{
Acoustic Noise Radiated by PWM-Controlled Induction Machine Drives
}

\author{
W. C. Lo, Member, IEEE, C. C. Chan, Fellow, IEEE, Z. Q. Zhu, Member, IEEE, Lie Xu, David Howe, and \\ K. T. Chau, Member, IEEE
}

\begin{abstract}
This paper investigates the acoustic noise radiated from two nominally identical induction motors when fed from sinusoidal, and asymmetric regular sampling subharmonic and space-vector pulsewidth modulation (PWM) converters. The theory for analyzing the noise spectrum is developed further to account for the interaction between the motor and the drive. It is shown that manufacturing tolerances can result in significant differences in the noise level emitted from nominally identical motors, and that mechanical resonances can result in extremely high noise emissions. Such resonances can be induced by stator and rotor slot air-gap field harmonics due to the fundamental component of current, and by the interaction between the airgap field harmonics produced by the fundamental and the PWM harmonic currents. The significance of the effect of PWM strategy on the noise is closely related to the mechanical resonance with vibration mode order zero, while the PWM strategy will be critical only if the dominant cause of the emitted noise is the interaction of the fundamental air-gap field and PWM harmonics.
\end{abstract}

Index Terms-Acoustic noise measurement, induction motors, pulsewidth modulated inverters.

\section{NOMENCLATURE}

$b(\theta, t) \quad$ Air-gap flux density harmonic components.

$f_{1} \quad$ Operating frequency of the motor.

$f_{k} \quad$ Frequency of $k$ th current harmonics.

$f_{s} \quad$ Switching frequency.

$k, k$ th Current harmonic order.

$m \quad$ Mode order.

$p \quad$ Number of pole pairs of the motor.

$F(\theta, t) \quad$ Air-gap MMF.

$F_{1} \quad$ Fundamental air-gap MMF wave produced by the fundamental current.

$F_{k} \quad$ First-order MMF harmonics due to the $k$ th current harmonics.

$F_{\mu} \quad$ Higher order MMF harmonics produced by the fundamental current of the rotor $\mu=q_{2} Q_{r}+\nu, q_{2}=$ $0, \pm 1 \pm 2, \ldots$.

$F_{\nu} \quad$ Higher order MMF harmonics produced by the fundamental current of the stator $\nu=(6 q+1) p, q=$

Manuscript received June 23, 1999; revised January 22, 2000. Abstract published on the Internet April 21, 2000.

W. C. Lo, C. C. Chan, and K. T. Chau are with the Department of Electrical and Electronic Engineering, University of Hong Kong, Hong Kong (e-mail:wclo@eee.hku.hk).

Z. Q. Zhu and D. Howe are with the Department of Electronic and Electrical Engineering, University of Sheffield, Sheffield, S1 3JD, U.K.

L. Xu was with the Department of Electronic and Electrical Engineering, University of Sheffield, Sheffield, S1 3JD, U.K. He is now with the Inter-University Glasgow-Strathclyde Centre for Economic Renewable Power Delivery, Glasgow, G1 1XW, U.K

Publisher Item Identifier S 0278-0046(00)06821-0.
$0, \pm 1 \pm 2, \cdots$ in which the slot MMF harmonics are $\nu=q_{1} Q_{s}+p, q_{1}=0, \pm 1 \pm 2, \ldots$.

$F_{\mu k} \quad$ Higher order MMF harmonics produced by the $k$ th current harmonics of the rotor.

$F_{\nu k} \quad$ Higher order MMF harmonics produced by the $k$ th current harmonics of the stator.

$Q_{s} \quad$ Slot number of stator.

$Q_{r} \quad$ Slot number of rotor.

$s_{k} \quad$ Slip of the $k$ th time harmonic.

$\varphi_{m 0} \quad$ Phase angle of the vibration force density wave for mode order $m$.

$\lambda(\theta, t) \quad$ Air-gap permeance.

$\mu, \mu$ th $\quad$ Rotor slot harmonics.

$\nu, \nu$ th $\quad$ Stator slot harmonics.

$\sigma_{r}(\theta, t) \quad$ Vibration force density wave.

$\sigma_{m} \quad$ Amplitude of the vibration force density wave for mode order $m$.

$\omega_{1} \quad$ Fundamental angular frequency of the supply.

$\omega_{k} \quad$ Angular frequency of the $k$ th current harmonic.

$\omega_{m} \quad$ Angular frequency for mode order $m$.

$\omega_{r} \quad$ Mechanical angular frequency of the rotor.

$\omega_{\mu k} \quad$ Harmonic angular frequency for $F_{\mu k}$.

$\omega_{\mu} \quad$ Harmonic angular frequency for $F_{\mu}$.

$\Lambda_{0}$, Amplitudes of permeance related to the average air-gap length.

$\Lambda_{k_{1}} \quad$ Amplitudes of permeance related to the stator slotting.

$\Lambda_{k_{2}} \quad$ Amplitudes of permeance related to the rotor slotting.

\section{INTRODUCTION}

$\mathbf{T}$ HE acoustic noise radiated by induction machines increases when they are operated from nonsinusoidal power supplies, such as quasi-square waveform and pulsewidth modulation (PWM) converters [1], [2]. This can be of considerable significance in certain applications, for example, when they are employed as traction machines in the drivetrain of electric vehicles. Since the total harmonic distortion is then increased, the emitted acoustic noise level can become unacceptable. However, although the effect of nonsinusoidal supplies on the acoustic noise has been addressed extensively [3]-[9], and various PWM strategies, such as natural sampling, asymmetrical regular sampling, harmonic elimination, optimal, space vector, and random PWM, have been developed primarily to reduce harmonic distortion and improve efficiency, their effect on noise emissions has been largely restricted to a consideration of the 
switching noise of the drive, with relatively little consideration of their interaction with the machines.

In order to compare the relative merits of different PWM strategies, an equivalent $A$-weighted total harmonic distortion (THD) is introduced for noise estimation [3], viz., $\mathrm{THD}_{v}=\sqrt{\sum\left(A_{n} V_{n} / V_{1}\right)^{2}} 100 \%$ or $\mathrm{THD}_{i}=\sqrt{\sum\left(A_{n} I_{n} / I_{1}\right)^{2}} 100 \%$ where $V_{1}, I_{1}$, and $V_{n}$, $I_{n}$ are the fundamental and harmonic components of the output voltage and current, and $A_{n}$ is the $A$-weighted curve accounting for the nonlinear response of the human ear to the sound pressure level. For conventional THD $A_{n}=1$, which is appropriate only in relation to a consideration of system losses, and is not appropriate when estimating the magnetic noise caused by harmonic components. Clearly, the equivalent $A$-weighted THD accounts for: 1) the harmonic voltage/current spectrum and 2) the acoustic behavior of the human ear. However, it still cannot account for mechanical resonances.

Both harmonic-eliminated and optimal PWMs with minimal harmonic content often result in reduced losses as well as a lower acoustic noise level [3]. However, as mentioned above, it is important to consider the $A$-weighted sound pressure level both for the switching harmonics and the natural frequencies. Bearing in mind that the current harmonics of a PWM-controlled drive are usually rich, while the relationship between the acoustic noise and current harmonics is nonlinear, both techniques may be very effective for a specific machine under constant speed operation, and they are not generally applicable and also difficult to realize for variable-speed drives.

Random PWM [4] disperses the tonal spectrum of a fixed-frequency switching PWM inverter drive into a wide-band atonal noise. Hence, it significantly reduces the subjective annoyance associated with the acoustic noise emitted from an inverter driven motor. The overall sound pressure level is largely unchanged with random switching, with any reduction arising from the nonexcitation of resonances which might previously be excited with fixed frequency switching [5]. In general, if a motor is operated from a random PWM converter: 1) the benefits will be insignificant if the motor noise is dominated by a resonance excited by the fundamental component of current; 2) the noise can be reduced significantly if the motor exhibits a resonance which is excited by the force due to the interaction of the fundamental component of current and PWM subharmonics; and 3) the noise may be increased, since random PWM may increase the probability of inducing mechanical resonances. Alternatively, by using music PWM techniques, otherwise arbitrary noise is converted into selected information, such as music, alarm, etc. Since the corresponding vibration and noise frequency will be centered around $2 f_{s}$ and $f_{s}$, the switching frequency $f_{s}$ should be equal to either the desired frequency or half of this frequency [6] in order to generate magnetic noise with the desired music frequency. However, since there are usually lots of noise components, in addition to those produced by the dominant current harmonics, the music effect is usually less than satisfactory.

One simple method to reduce the effect of acoustic noise is to use ultrasonic switching [7] which effectively shifts the PWM harmonics out of the human audible frequency range $(>20$ $\mathrm{kHz}$ ). It is, therefore, most desirable. However, current power semiconductor switch technology limits this to low power levels using MOSFETs, since high switching frequency results in high switching loss in insulated gate bipolar transistors (IGBTs) and MOSFET-controlled thyristors (MCTs). A resonant converter facilitates ultrasonic frequency switching and thereby provides another potential for acoustic noise reduction [1]. Another method of reducing the noise from PWM controlled drives is to make sinusoidal output waveforms using filters which are connected to inverter output terminals [8]. However, although they are always beneficial, not all harmonics can be sufficiently eliminated since the magnetic noise is caused by the least residual harmonics, which cannot be absorbed by output filters.

It is interesting to note that by using two small inverters to drive a large induction machine equipped with two separate stator windings the noise and current ripple can be reduced by triggering two PWM inverters with inverse pulses so that the switching frequency harmonic voltages are in antiphase. In this way, the noise level can be reduced [9]. However, the method is really only practical for very high power induction machine drives.

This paper describes an investigation of the influence of PWM strategies on the acoustic noise emitted by two nominally identical, mass produced three-phase six-pole $50-\mathrm{Hz} / 60-\mathrm{Hz}$ induction machines. Firstly, the noise variation from the machines is measured, and the noise and vibration spectra are analyzed for $50-\mathrm{Hz} / 60-\mathrm{Hz}$ sinusoidal waveform operation. Asymmetric regular sampling and space-vector PWM (SVPWM) are then compared, and the effect of the operating speed and switching frequency is investigated.

\section{NOISE SPECTRUM ANALYSIS}

The electromagnetically generated acoustic noise spectrum can be determined from the radial vibration force waves, which are usually evaluated analytically by the Maxwell stress method by neglecting the circumferential component of the air-gap flux density. Although skewing affects the amplitudes of the resulting noise components it does not affect the corresponding frequencies, i.e., the frequency spectrum is the same for both skewed and unskewed machines. Therefore, assuming an unskewed machine, the general form of the vibration force density wave, $\sigma_{r}(\theta, t)$, is given by

$$
\sigma_{r}(\theta, t)=b^{2}(\theta, t) / 2 \mu_{0}=\sum \sigma_{m} \cos \left(m \theta-\omega_{m} t-\varphi_{m 0}\right)
$$

$$
\begin{array}{ll}
\text { where } & =4 \pi \times 10^{-7} \\
\mu_{0} & \text { mode order; } \\
m & \text { angular frequency; } \\
\omega_{m} & \text { amplitude and phase angle of the force wave, } \\
\sigma_{m}, \varphi_{m 0} & \text { respectively. }
\end{array}
$$

The mode orders and angular frequencies can be computed from the orders and angular frequencies of the air-gap flux-density harmonic components, $b(\theta, t)$. In [2], the frequency spectra are calculated directly from (1). However, although it is entirely general, it gives little insight regarding the dominant components and the associated vibration modes. In this paper, these are derived analytically. 
As an approximation, $b(\theta, t)$ may be deduced from the product of the airgap mmf, $F(\theta, t)$, and the air-gap permeance, $\lambda(\theta, t)$, i.e.,

$$
b(\theta, t)=F(\theta, t) \lambda(\theta, t) .
$$

The MMF wave in a PWM-inverter-fed three-phase induction motor can be obtained in a similar way to the machines fed from sinusoidal power supply [10] and can be expressed as

$$
\begin{aligned}
& F(\theta, t) \\
& =F_{1} \cos \left(p \theta-\omega_{1} t-\varphi_{1}\right)+\sum F_{k} \cos \left(p \theta-\omega_{k} t-\varphi_{k}\right)+\sum_{\nu} \\
& \quad \cdot F_{\nu} \cos \left(\nu \theta-\omega_{1} t-\varphi_{\nu}\right)+\sum F_{\mu} \cos \left(\mu \theta-\omega_{\mu} t-\varphi_{\mu}\right)+\sum_{k \neq 1} \\
& \quad \cdot F_{\nu k} \cos \left(\nu \theta-\omega_{k} t-\varphi_{\nu k}\right)+\sum_{k \neq 1} F_{\mu k} \cos \left(\mu \theta-\omega_{\mu k} t-\varphi_{\mu k}\right)
\end{aligned}
$$

where

$p$ number of pole pairs:

$\omega_{1}$ fundamental angular frequency of the supply;

$\omega_{k}=k \omega_{1}$ angular frequency of the $k$ th current harmonic.

The first term is the fundamental air-gap MMF wave produced by the fundamental current, and the second term represents firstorder MMF harmonics due to current harmonics. The third and fourth terms are higher order MMF harmonics produced by the fundamental current of the stator and the rotor, respectively, while the fifth and sixth terms are higher order MMF harmonics produced by the current harmonics of the stator and the rotor, respectively. For a three-phase induction motor, $\nu=(6 q+1) p$, $q=0, \pm 1 \pm 2, \cdots$ in which the slot MMF harmonics are $\nu=$ $q_{1} Q_{s}+p, q_{1}=0, \pm 1 \pm 2, \cdots$ whilst $\mu=q_{2} Q_{r}+\nu$, in which $\nu=p$ is usually the most important, and $q_{2}=0, \pm 1 \pm 2, \cdots$ $Q_{s}$ and $Q_{r}$ being the stator and rotor slot numbers, respectively. The harmonic angular frequencies are [10]

$$
\omega_{\mu k}=\omega_{k}\left[1+q_{2} \frac{Q_{r}}{p}\left(1-s_{k}\right)\right]
$$

when $k=1$,

$$
\omega_{\mu}=\omega_{1}\left[1+q_{2} \frac{Q_{r}}{p}\left(1-s_{1}\right)\right]
$$

where $s_{k}$ is the slip of the $k$ th time harmonic.

$$
s_{k}=\left(\omega_{k}-p \omega_{r}\right) / \omega_{k}
$$

where $\omega_{r}$ is the mechanical angular frequency of the rotor. Clearly, $s_{1} \approx 0$ at no load for the fundamental MMF, $k=1$, while $s_{k} \approx 1$ for time harmonics and, hence, $\omega_{\mu k} \approx \omega_{k}$ for $k \neq 1$.

The air-gap permeance, accounting for stator and rotor slotting, can be expressed as [10]

$$
\begin{aligned}
\lambda & =\Lambda_{0}\left(1+\frac{\sum \lambda_{1}}{\Lambda_{0}}\right)\left(1+\frac{\sum \lambda_{2}}{\Lambda_{0}}\right) \\
& \approx \Lambda_{0}+\sum \lambda_{1}+\sum \lambda_{2}
\end{aligned}
$$

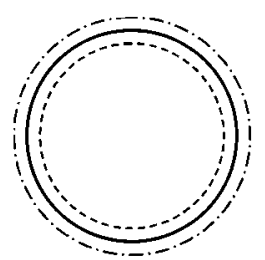

(a)

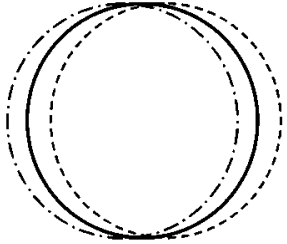

(b)

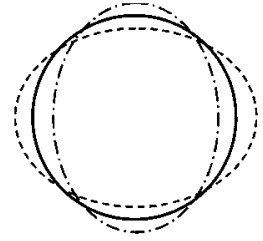

(c)
Fig. 1. Definition of vibration mode

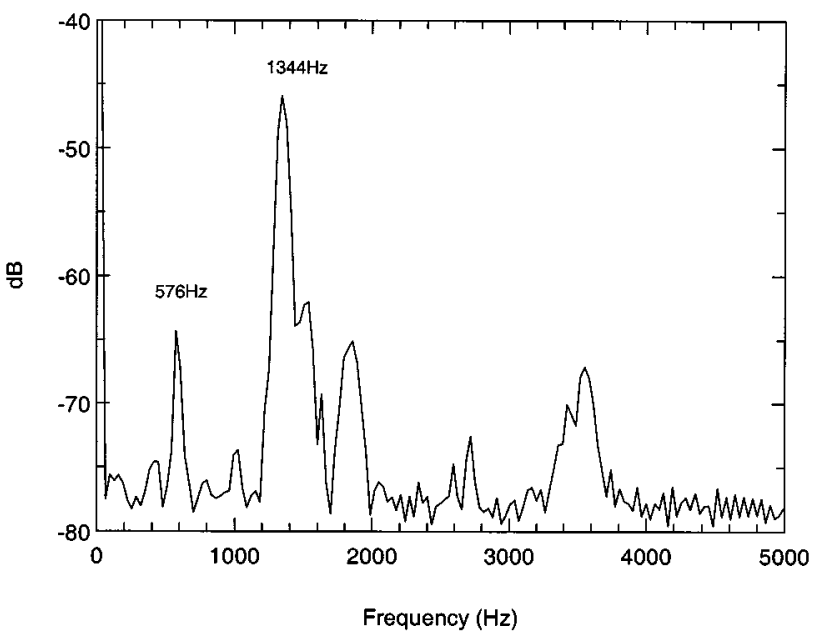

(a)

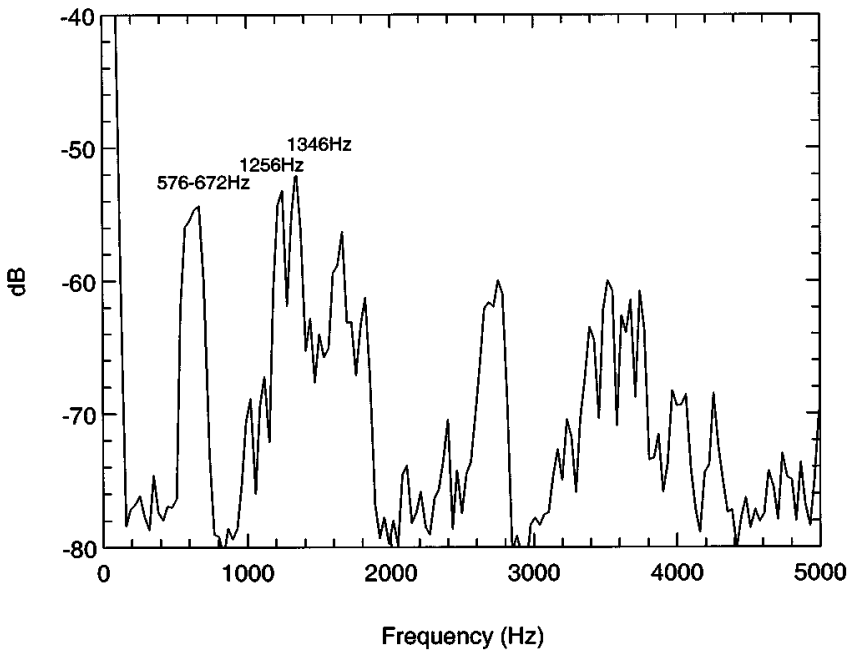

(b)

Fig. 2. Natural frequencies.

where

$$
\begin{aligned}
\sum \lambda_{1} & =\sum_{k_{1}=1,2, \cdots} \Lambda_{k_{1}} \cos k_{1} Q_{s} \theta \\
\sum \lambda_{2} & =\sum_{k_{2}=1,2, \ldots} \Lambda_{k_{2}} \cos k_{2} Q_{r}\left[\theta-\frac{\omega_{1}}{p}(1-s) t\right]
\end{aligned}
$$

where $\Lambda_{0}, \Lambda_{k_{1}}$, and $\Lambda_{k_{2}}$ are the amplitudes of permeance related to the average air-gap length, and the stator and rotor slotting, respectively.

The air-gap flux density and the radial vibration force waves can be deduced from (1) to (6). It is well known that the vibration 


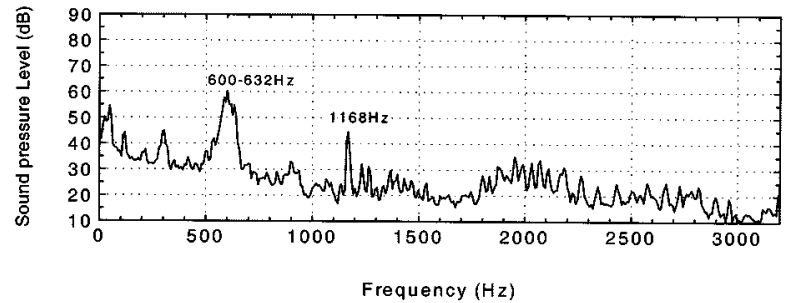

(a)

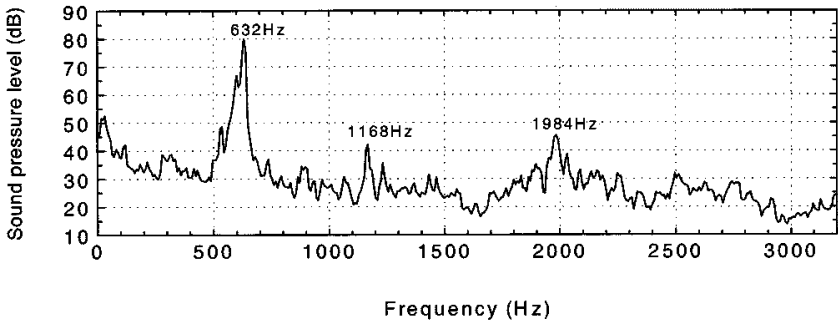

(b)

Fig. 3. Noise spectra when operated from 50-Hz/400-V sinusoidal supply

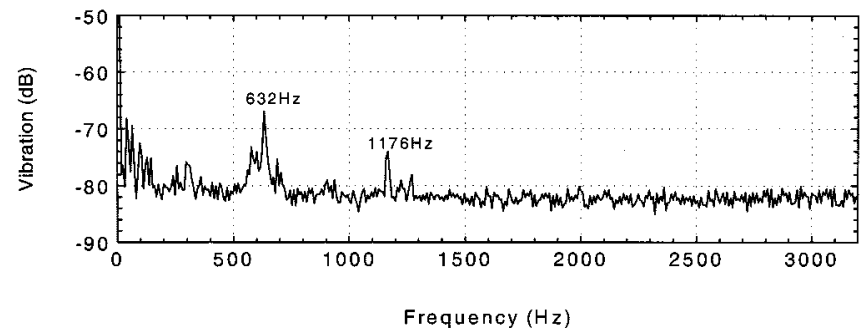

(a)

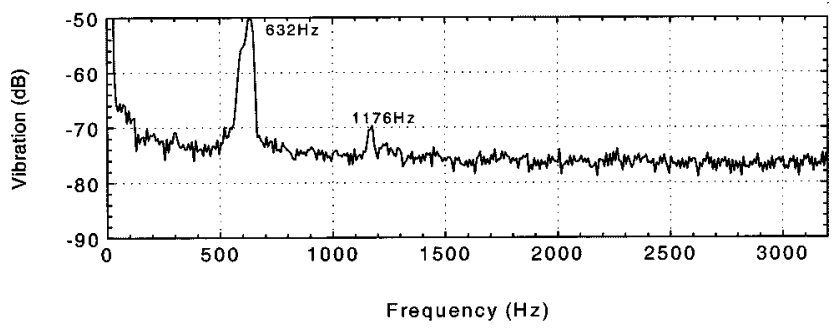

(b)

Fig. 4. Vibration spectra when operated from 50-Hz/400-V sinusoidal supply

force waves, the vibration response, and the radiated acoustic noise have identical frequency spectra. The important vibration forces are produced by the interactions between the following pairs of flux density waveforms (for simplicity, they are represented only by their amplitudes):

1) the stator and rotor mmf and permeance slot field harmonics, $\left\langle\left(F_{\nu} \Lambda_{0}\right)+\left(F_{1} \Lambda_{k_{1}}\right),\left(F_{\mu} \Lambda_{0}\right)+\left(F_{1} \Lambda_{k_{2}}\right)\right\rangle$, viz., the interaction between the resultant stator mmf and permeance slot harmonics and the resultant rotor MMF and permeance slot harmonics;

2) the first-order field harmonics produced by the fundamental current and its harmonics, $\left\langle\left(F_{k_{1}} \Lambda_{0}\right),\left(F_{k_{2}} \Lambda_{0}\right)\right\rangle$, in which $k_{1}=1$ or $k_{2}=1$ represents the fundamental component;
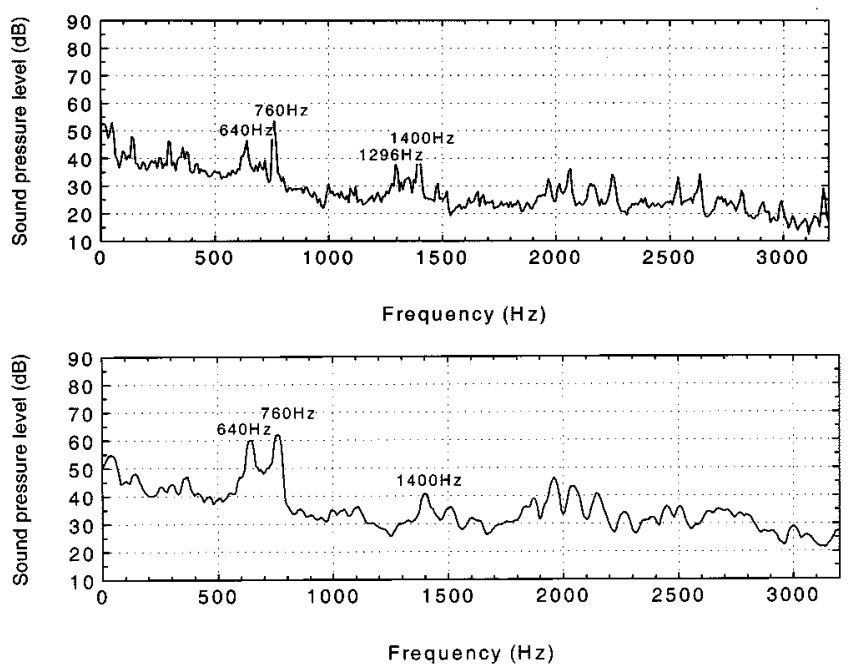

Fig. 5. Noise spectra when operated from $60-\mathrm{Hz} / 480-\mathrm{V}$ sinusoidal supply
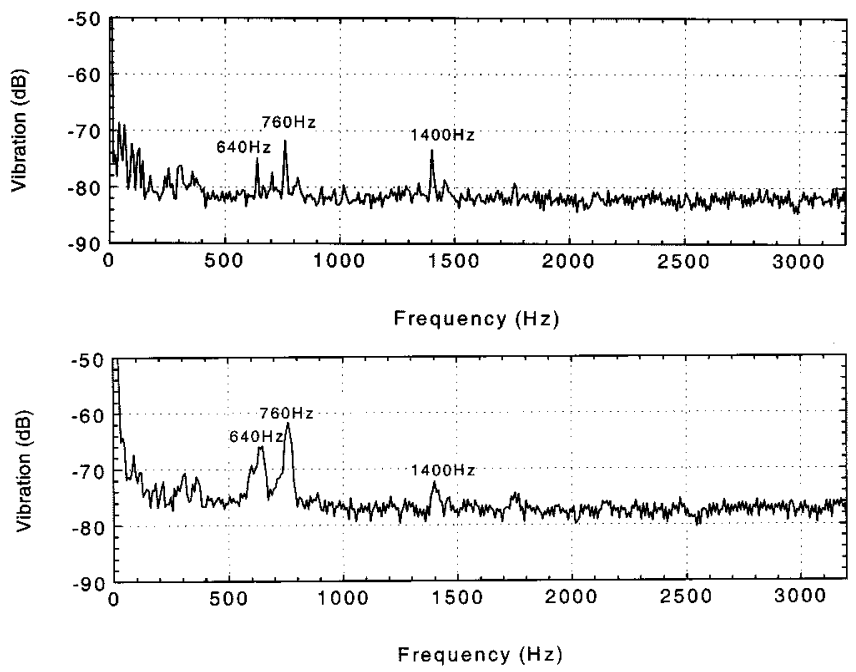

Fig. 6. Vibration spectra when operated from $60-\mathrm{Hz} / 480-\mathrm{V}$ sinusoidal supply

3) the permeance field harmonics and the MMF field harmonics associated with the current harmonics, e.g.,

$$
\begin{array}{ll}
\left\langle\left(F_{1} \Lambda_{k_{2}}\right),\left(F_{\nu k} \Lambda_{0}\right)\right\rangle, & \left\langle\left(F_{1} \Lambda_{k_{1}}\right),\left(F_{\mu k} \Lambda_{0}\right)\right\rangle, \\
\left\langle\left(F_{1} \Lambda_{k_{1}}\right),\left(F_{\nu k} \Lambda_{0}\right)\right\rangle, & \left\langle\left(F_{1} \Lambda_{k_{2}}\right),\left(F_{\mu k} \Lambda_{0}\right)\right\rangle ; \\
\left\langle\left(F_{k} \Lambda_{k_{2}}\right),\left(F_{\nu} \Lambda_{0}\right)\right\rangle, & \left\langle\left(F_{k} \Lambda_{k_{1}}\right),\left(F_{\mu} \Lambda_{0}\right)\right\rangle, \\
\left\langle\left(F_{k} \Lambda_{k_{1}}\right),\left(F_{\nu} \Lambda_{0}\right)\right\rangle, & \left\langle\left(F_{k} \Lambda_{k_{2}}\right),\left(F_{\mu} \Lambda_{0}\right)\right\rangle ; \\
\left\langle\left(F_{\nu} \Lambda_{0}\right),\left(F_{\mu k} \Lambda_{0}\right)\right\rangle, & \left\langle\left(F_{\nu} \Lambda_{0}\right),\left(F_{\nu k} \Lambda_{0}\right)\right\rangle, \\
\left\langle\left(F_{\mu} \Lambda_{0}\right),\left(F_{\mu k} \Lambda_{0}\right)\right\rangle, & \left\langle\left(F_{\mu} \Lambda_{0}\right),\left(F_{\nu k} \Lambda_{0}\right)\right\rangle ; \\
\left\langle\left(F_{1} \Lambda_{k_{2}}\right),\left(F_{k} \Lambda_{k_{1}}\right)\right\rangle, & \left\langle\left(F_{1} \Lambda_{k_{1}}\right),\left(F_{k} \Lambda_{k_{2}}\right)\right\rangle .
\end{array}
$$

The vibration forces in 2) and 3) are associated only with nonsinusoidal current waveforms.

When rotor eccentricity and magnetic circuit saturation are neglected, the most important frequencies of the radial electromagnetic forces can be derived from (1)-(6) and are given by the following.

a) Included are vibration forces produced by the interaction of the $\mu$ th stator and the $\nu$ th rotor slot harmonics, i.e., the 



Fig. 7. Voltage, current, and noise for subharmonic PWM (SPWM), $f_{1} / f_{s}=50 / 1350 \mathrm{~Hz}, M=0.95$.

components in 1). In this case, the dominant force components are usually associated with the fundamental current and, hence, they are identical to those when operated from a sinusoidal supply. The frequencies and modes are given by

$$
\begin{aligned}
f=f_{1} & {\left[q_{2} \frac{Q_{r}}{p}\left(1-s_{1}\right)+2\right], } \\
& \quad \text { or vibration mode order } m^{+}=\mu+\nu, \\
f=f_{1} & {\left[q_{2} \frac{Q_{r}}{p}\left(1-s_{1}\right)\right], } \\
& \quad \text { or vibration mode order } m^{-}=\mu-\nu
\end{aligned}
$$

where $q_{2}=0, \pm 1, \pm 2, \ldots, f_{1}$ is the operating frequency of the motor. The higher the harmonic order $\mu$ and $\nu$, the smaller the amplitudes of the magnetic field harmonics, while the lower the mode orders the more important the resulting vibration force. The important electromagnetic noise components under all load conditions are related to those force components having the lowest mode orders, i.e., $m=0,2,3,4$ (see Fig. 1). However, due to corresponding high field harmonic orders and higher natural frequencies, the noise components associated with $m=0$, Fig. 1(a), are usually less important than those associated with $m=2,3,4$ when operated from a sinusoidal supply. b) Also included are vibration forces due to the interaction of the first-order field harmonics produced by the fundamental current and its harmonics, i.e., component 2). They are large in amplitude, and the frequencies are located around multiples of the switching frequency, while the vibration modes are zero, i.e.,

$$
f=\left|\left( \pm f_{k_{1}}\right)-\left( \pm f_{k_{2}}\right)\right| \text { and } m=0
$$

where "+" and "-" correspond to forward and backward rotation of the field harmonics, respectively, and $f_{k}$ is the frequency of $k$ th current harmonics. For example, for natural PWM, the voltage and current harmonics are $f_{k}=$ $n_{1} f_{s} \pm n_{2} f_{1}$ where if $n_{1}$ is an odd integer $n_{2}$ will be an even integer, while if $n_{1}$ is an even integer $n_{2}$ will be an odd integer, i.e., $f_{k}=f_{s} \pm 2 f_{1}, f_{s} \pm 4 f_{1}, \ldots$ and $2 f_{s} \pm f_{1}, 2 f_{s} \pm 3 f_{1}, \ldots$ etc. where $f_{s}$ is the switching frequency. However, in this case, normally the interaction between the fundamental air-gap field and the first-order field harmonics produced by the current harmonics is the most important, and the frequencies are given by

$$
f=\left|\left( \pm f_{k}\right)-f_{1}\right| \text { and } m=0
$$

c) Vibrations due 3) are also sometimes important, particularly on full load and when the vibration modes are low, 

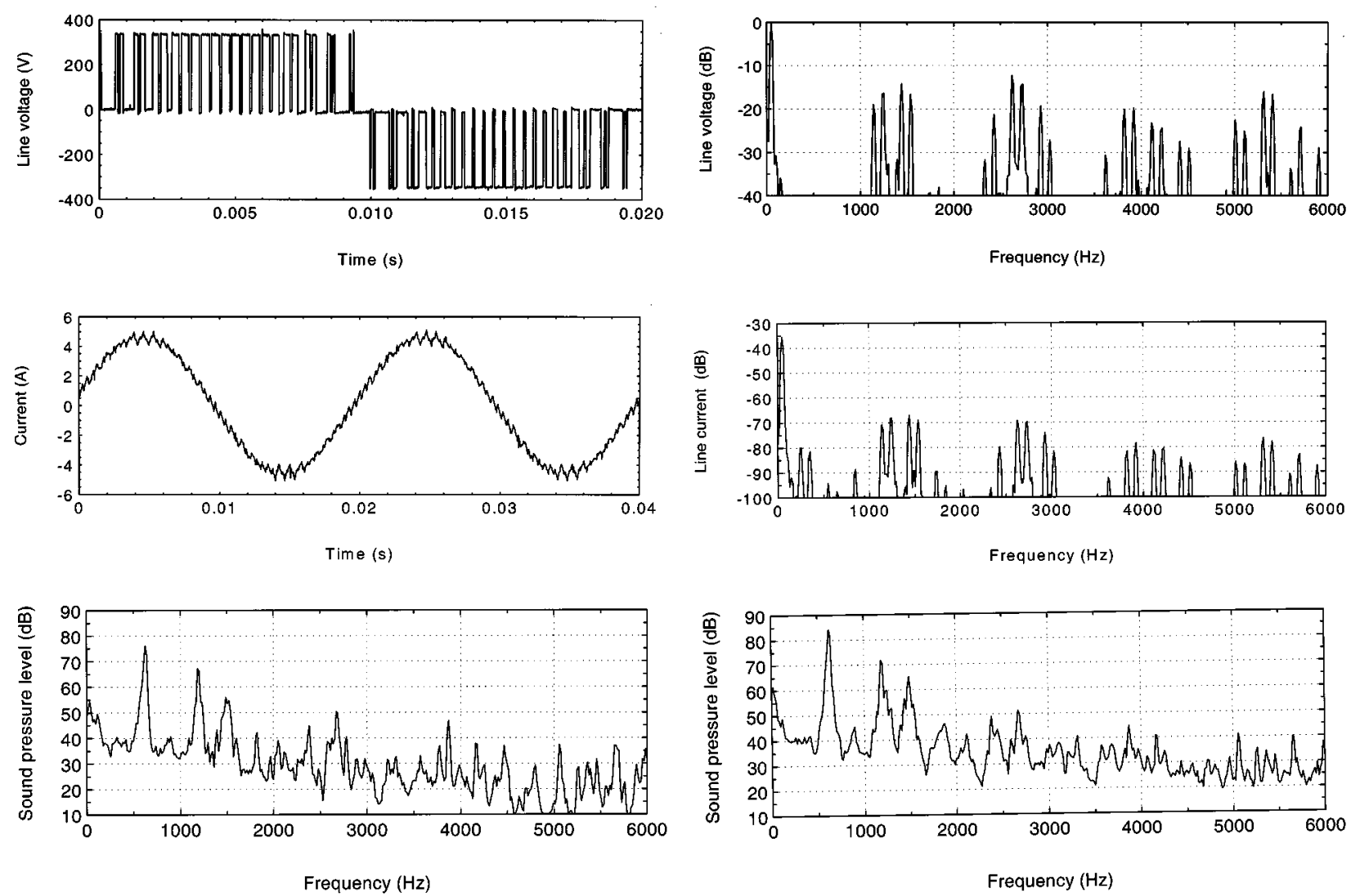

Fig. 8. Voltage, current, and noise for SVPWM, $f_{1} / f_{s}=50 / 1350 \mathrm{~Hz}, M=0.95$.

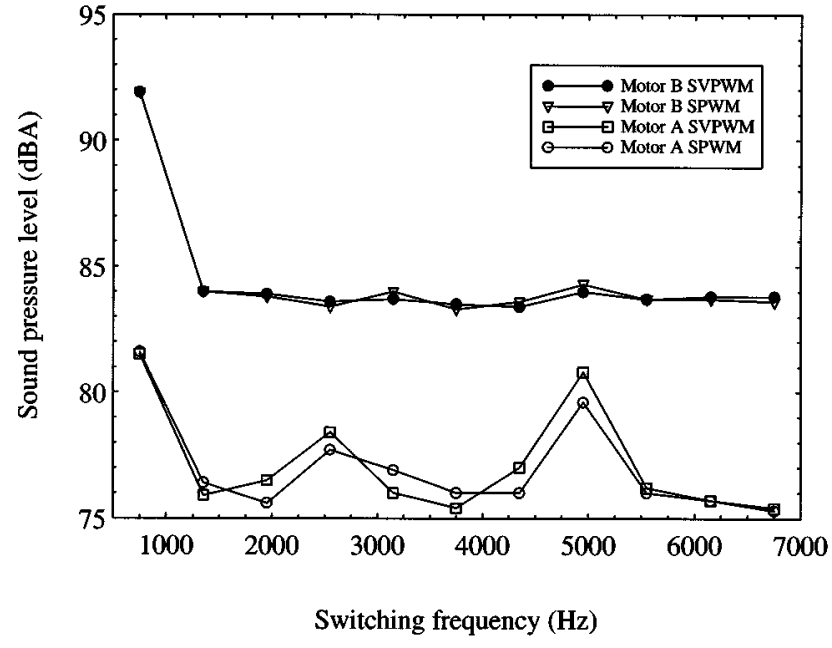

ANALYSIS OF NOISE SPECTRA (RESOLUTION FOR FREQUENCY READING IS 16 Hz; “+” = FORWARD ROTATING; “-” = BACKWARD ROTATING)

\begin{tabular}{lc|c|c|c}
\hline & $\begin{array}{c}\text { Noise } \\
(\mathrm{Hz})\end{array}$ & $\begin{array}{c}\text { Vibration } \\
\text { Mode }\end{array}$ & $\begin{array}{c}\text { SVPWM } \\
(\mathrm{Hz})\end{array}$ & $\begin{array}{c}\text { SPWM } \\
(\mathrm{Hz})\end{array}$ \\
\hline 1 & 640 & 2 & 50, stator/rotor slotting & 50 , stator/rotor slotting \\
\hline 2 & 1184 & 0 & $(50,1136)$ & \\
& & & $\left(50,1232^{+}\right)$ & $\left(50,1232^{+}\right)$ \\
\hline 3 & 1488 & 0 & $\left(50,1440^{-}\right)$ & $\left(50,1440^{-}\right)$ \\
& & & $\left(50,1536^{+}\right)$ & \\
\hline 4 & 2672 & 0 & $(50,2624)$ & $(50,2624)$ \\
\hline 5 & & & $\left(50,2736^{+}\right)$ & $\left(50,2736^{+}\right)$ \\
\hline 6 & 4856 & 0 & $\left(50,3824^{+}\right)$ & $(50,3824)$ \\
& & & $\left(50,3920^{+}\right)$ & $\left(50,3920^{+}\right)$ \\
\hline
\end{tabular}

Fig. 9. Variation of noise with SVPWM and SPWM $\left(f_{1}=50 \mathrm{~Hz}, M=\right.$ $0.95)$.

e.g., 0 or 2 . It can be shown that the corresponding frequencies are given by

$$
f=\left\{\begin{array}{l}
\left|\left( \pm f_{k}\right)-f_{1}\right| \\
\left|f_{k} \pm\left(f_{1}+q_{2} \frac{Q_{r}}{p}(1-s) f_{1}\right)\right|, \quad \text { when } m=0,2
\end{array} .\right.
$$

It will be shown that the noise sources a) and b) are the most important components on no load. However, as the load

increases, the noise source c) also becomes important. If the frequencies and modes of the important vibration forces coincide with mechanical natural frequencies and modes, the machine will emit a high acoustic noise. Clearly, the acoustic noise radiated from PWM-controlled variable-speed induction machine drives depends on various electromagnetic, mechanical, acoustic, and control parameters, e.g., 1) the pole number, the stator and rotor slot number combination; 2) the motor operating frequency; 3) the PWM switching frequency; and 4) mechanical natural frequencies and vibration modes of the system. 
It is worth emphasizing that, although natural frequencies and vibration forces in a PWM-controlled induction machine are both very rich, (8) shows that the vibration mode of the most important excitation forces associated with PWM harmonics is zero, which is a pulsating mode [see Fig. 1(a)]. Hence, it is possible to select appropriate PWM strategies to avoid mechanical resonances.

\section{ANalysis of Noise When Machines Fed From SINUSOIDAL POWER SUPPLY}

In the following analysis, the motors had six poles and a 36 stator slot/32 rotor slot combination. A dynamic signal analyzer, HP35660A, and a precision sound level meter, B\&K 2221, were used to measure the natural frequencies and acoustic noise spectra on no load. The influence of the load condition will be reported in due course. The noise measurements were made in an anechoic chamber, all the measurements being made with the microphone placed $200 \mathrm{~mm}$ from the outer surface of the motors. The noise problem was identified as electromagnetic, rather than aerodynamic or mechanical, as will be evidenced from the subsequent analysis.

The natural frequencies of both Motors A and B were obtained by applying an impulse force radially using a force hammer onto the stator of the whole machine assembly and measuring the vibration response using an accelerometer; the details of the test method can be found in [11]. The results are shown in Fig. 2. It will be seen that they exhibit a number of natural frequencies, which are associated with different vibration modes. However, as a result of normal manufacturing tolerances, the lowest natural frequency for Motor B is around $576-672 \mathrm{~Hz}$ (wide peak), while that for Motor A is $576 \mathrm{~Hz}$ (narrow peak). The mode order is 1 [see Fig. 1(b)], which is essentially an oscillating vibration of the stator with reference to the rotor. It was shown [11] that when the frequency of the excitation force is close to the natural frequency of such a vibration mode a mechanical resonance will occur even when the mode order of the excitation force is different. In addition, there is another natural frequency around $9-10 \mathrm{kHz}$ associated with vibration mode zero, which, as will be shown later, is the most important regarding the noise due to the vibration forces produced by the interaction of the fundamental air-gap field and the first-order field harmonics resulting from the current harmonics.

When they were run on no load from a $50-\mathrm{Hz} / 400-\mathrm{V}$ power supply, the noise spectra of both Motors A and B were dominated by the $640-\mathrm{Hz}$ frequency component (note that the frequency may exhibit a small error due to the discretized nature of the spectrum). See Fig. 3. According to the theory which was presented earlier, this is due to the radial vibration force produced by the interaction between the first stator and rotor slot harmonics (see Fig. 4) for which the corresponding vibration mode order is 2 [see Fig. 1(c)]. There is another component at $1176 \mathrm{~Hz}$ with mode order 2, which is due to the interaction between the second stator and rotor slot harmonics, although it is not as prominent as the $640-\mathrm{Hz}$ frequency component. Since the frequency of the main radial excitation force when operated from the $50-\mathrm{Hz}$ power supply is $640 \mathrm{~Hz}$, it is clear from



(a)

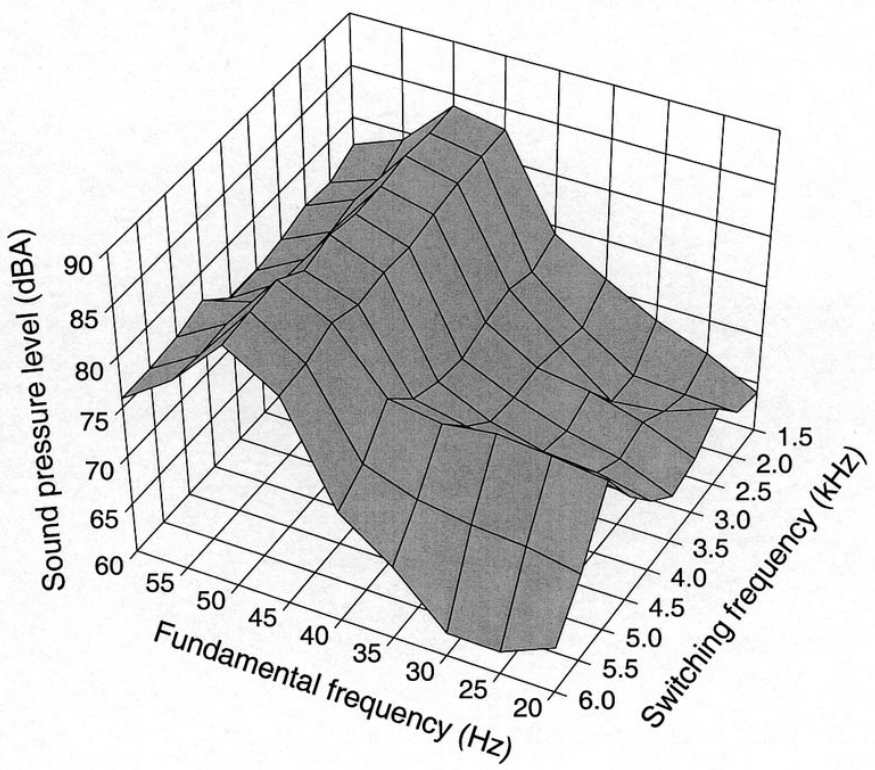

(b)

Fig. 10. Variation of noise with fundamental operating frequency and switching frequency when supplied from an SVPWM converter under constant $V / f_{1}$.

Figs. 2(b), 3(b), and 4(b) that it coincides with a mechanical resonance for Motor B. Although the vibration mode order corresponding to the excitation force at this frequency is 2 , while the vibration mode order corresponding to the lowest natural frequency is 1 [ see Fig. 1(b)] (i.e. they are different), it can still cause resonance as long as the natural vibration mode order is 1 as mentioned earlier. In contrast, Motor A does not exhibit a mechanical resonance since its lowest natural frequency is below $600 \mathrm{~Hz}$. It should be noted that the above-mentioned mode orders are referred to as vibration mode orders, not force mode orders. Vibration response can be measured easily by using accelerometers, as compared with forces.

When the motors were supplied from a $60-\mathrm{Hz} / 480-\mathrm{V}$ power supply, the dominant frequency components in the noise spectra for both Motors A and \& B shift to 760 and $1400 \mathrm{~Hz}$, although their vibration modes remain the same (see Figs. 5 and 6). Since 

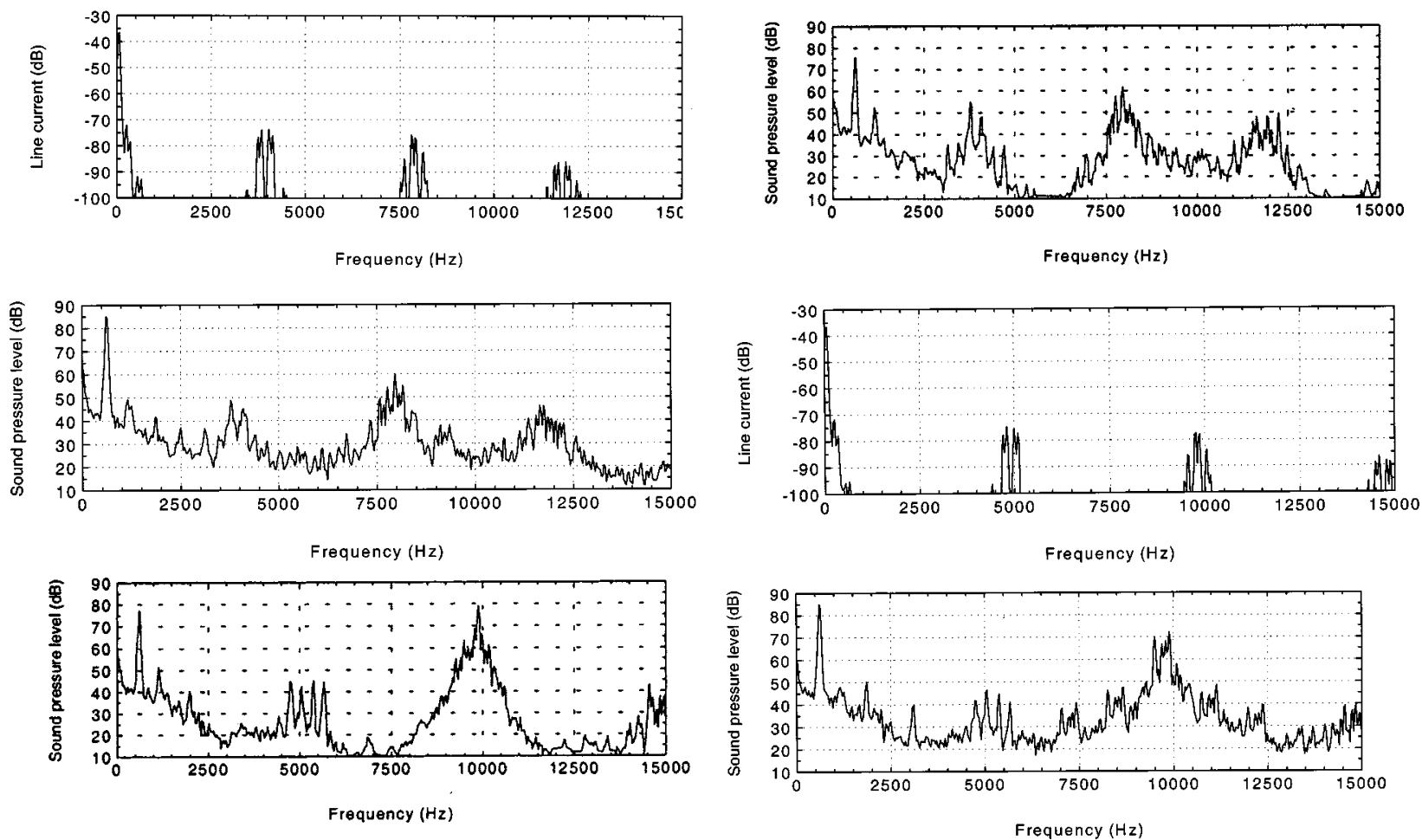

Fig. 11. Current and noise spectra.

the frequency of the main radial excitation force is now $760 \mathrm{~Hz}$, there is no resonance and, hence, the total noise level is lower. However, there is another excitation frequency of 640-660 Hz with vibration mode order 4 , which is also produced by the interaction between the first stator and rotor slot harmonics, and which is now coincident with the lowest natural frequency (see Figs. 5 and 6). However, since the mode order of the excitation force is high, the resonance is not so severe, and the associated noise level is much lower. Therefore, the motors were much quieter when they were run from a $60-\mathrm{Hz}$ supply rather than $50 \mathrm{~Hz}$.

\section{InVESTIGATION OF ACOUSTIC NOISE IN PWM-CONTROLLED INDUCTION MACHINE DRIVES}

In this section, the acoustic noise when the motors are supplied from asymmetric regular sampling SPWM [12] and SVPWM [13] converters is compared, and the effect of the operating speed and the switching frequency are investigated.

In order to investigate the effect of PWM current harmonics on the radiated acoustic noise, the motor was supplied from a TMS320C50 digital-signal-processor-controlled MOSFET inverter for various operating frequencies $f_{1}$, the modulation index being varied to maintain a constant V/f ratio. Figs. 7 and 8 show the line-line voltage and current waveforms and their corresponding spectra for a specific ratio of the fundamental to switching frequency $f_{1} / f_{s}$. It can be seen that, for SVPWM, both the voltage and current harmonics exhibit a slightly wider harmonic bandwidth than those for regular sampling asymmetrical SPWM, but the amplitudes are lower. However, measurements show that SVPWM results in almost the same noise spectra and noise level as SPWM (see Figs. 7 and 8). This is not surprising, since, although the difference between

the vibration forces, which are due to the interaction of the fundamental air-gap field and the first-order field harmonics produced by the current harmonics, is noticeable on a linear scale, the corresponding difference in the radiated acoustic noise level (in decibels) is insignificant. It is pertinent to note that, since SVPWM has a wider harmonic bandwidth than SPWM, it is more likely to induce a mechanical resonance and, indeed, a slightly higher acoustic noise can be observed in Fig. 9 around switching frequencies of 2.5 and $5 \mathrm{kHz}$, respectively, which cause mechanical resonances at the natural frequency around $10 \mathrm{kHz}$. However, it will be noted that for the resultant $A$-weighted sound pressure level these are only noticeable on Motor A, for which the frequencies of the vibration force produced by the stator and rotor slot harmonics do not coincide with a mechanical resonance. Fig. 9 also shows that, when supplied from both SPWM and SVPWM converters, Motor B still radiates much higher acoustic noise than Motor A, due to the resonance of Motor $\mathrm{B}$ at the $50-\mathrm{Hz}$ fundamental frequency. However, when the switching frequency is very low, the current harmonics and the acoustic noise increase very significantly for both motors, which were found to be almost as noisy as when they were operated from a quasi-square converter.

Applying the theory for the noise spectrum analysis, the dominant noise components can be identified. The results are summarized in Table I, where it can be seen that the dominant noise components for both SVPWM and SPWM are identical.

From (7), it can be shown that component 1 in Table I, i.e., $640 \mathrm{~Hz}$, is due to the radial vibration force produced by the interaction of the first order stator and rotor slot field harmonics associated with the $50-\mathrm{Hz}$ fundamental current, and that the corresponding vibration mode is 2 . As when the machines are fed from a sinusoidal power supply, this vibration force 
induces a mechanical resonance at the lowest natural frequency of $576-672 \mathrm{~Hz}$ for Motor B.

From (8), it can be shown that noise components ( 2 and 3 ), $4,(5$ and 6$)$ in Table I are due to vibration forces produced by the interaction of the fundamental air-gap field and the firstorder field harmonics produced by current harmonics around the switching frequency $f_{s}$ and its multiples, $2 f_{s}$ and $3 f_{s}$, as indicated by $(50,1136)$, etc. where 50 and $1136 \mathrm{~Hz}$ are the frequencies of the fundamental and harmonic current components. The corresponding vibration modes are all zero. In Table I, the symbols "+" and "-" indicate that the first-order field harmonic produced by this current harmonic is either forward and backward rotating. As shown in Table I, one noise component is usually produced by a pair of vibration forces.

In order to further illustrate the influence of the operating and switching frequencies, Fig. 10(a) and (b) shows the effect of varying both the motor speed $(20-60 \mathrm{~Hz})$ and the switching frequency $(1.5-6 \mathrm{kHz})$ of the SVPWM for Motors A and B. It is seen that Motor B still exhibits a mechanical resonance when operated at $50-\mathrm{Hz}$ fundamental frequency, when the effect of the PWM switching frequency becomes minimal. However, below this frequency the switching frequency has a significant effect on the emitted acoustic noise. Both Motors A and B also exhibit resonances around $10 \mathrm{kHz}$, induced by the excitation force produced by the fundamental air-gap field and the harmonics located around multiples of the switching frequency. As a result, the noise level is a maximum at 2.5- and $5-\mathrm{kHz}$ switching frequencies over the motor operating speed range, as mentioned earlier. However, this is only critical when there is no resonance solely due to the fundamental phase current.

Fig. 11 clearly illustrates the two types of mechanical resonances discussed above, namely: 1) the resonance of Motor B at $640 \mathrm{~Hz}$ when operated at $50 \mathrm{~Hz}$ fundamental frequency and 2) the resonance at $10 \mathrm{kHz}$ induced by PWM current harmonics at 2.5- and 5-kHz switching frequencies. Since Motors A and B have nominally identical electromagnetic characteristics, only the current spectra for Motor A are included in Fig. 11.

\section{CONCLusions}

The acoustic noise radiated from two nominally identical induction motors, fed from sinusoidal, asymmetric regular sampling SPWM and SVPWM converters, has been investigated theoretically and experimentally at different operating speeds and PWM switching frequencies. The following points have been shown.

1) Manufacturing tolerances can result in significant differences in the noise emitted from nominally identical motors.

2) Mechanical resonances can result in extremely high noise emissions, such resonances being induced by the fundamental current and PWM harmonics, both independently and in combination.

3) Analysis of the acoustic noise should consider the stator and rotor slot air-gap field harmonics due to the fundamental current, and the interaction between the air-gap field harmonics produced by the fundamental and PWM harmonic currents.

4) The vibration modes associated with the radial force produced by the interaction between the stator and rotor slot harmonics depend on the stator and rotor slot numbers and pole number, while the vibration modes are zero associated with the radial force produced by the interaction between the fundamental air-gap field and the first-order field harmonics produced by the current harmonics.

5) The significance of the effect of PWM switching frequency is closely related to the mechanical resonance whose vibration mode is zero.

6) The difference between the acoustic noise produced by the induction machine fed from SPWMs and SVPWMs is generally insignificant.

7) A motor which is noisy when fed from a sinusoidal supply will be still noisy when supplied from a PWM converter. However, the reverse may not be the case.

8) The PWM strategy is critical only when the emitted noise is dominated by the electromagnetic force resulting from the interaction of the fundamental air-gap field and PWM harmonics. Thus, the influence of the PWM strategy is likely to be minimal if a machine exhibits a resonance due to the fundamental current.

\section{REFERENCES}

[1] A. K. Wallance, R. Spee, and L. G. Martin, "Current harmonics and acoustic noise in AC adjustable-speed drives," IEEE Trans. Ind. Applicat., vol. 26, pp. 267-273, Mar./Apr. 1990.

[2] R. J. M. Belmans, L. D'hondt, A. J. Vandenput, and W. Geysen, "Analysis of the audible noise of three-phase squirrel-cage induction motors supplied by inverters," IEEE Trans. Ind. Applicat., vol. IA-23, pp. 842-847, Sept./Oct. 1987.

[3] S. Iida, Y. Okuma, S. Masukawa, S. Miyairi, and B. Bose, "Study on magnetic noise caused by harmonics in output voltages of PWM inverter," IEEE Trans. Ind. Electron., vol. 38, pp. 180-186, June 1991.

[4] T. G. Habetler and D. M. Divan, "Acoustic noise reduction in sinusoidal PWM drives using a randomly modulated carrier," IEEE Trans. Power Electron., vol. 6, pp. 356-363, July 1991.

[5] F. Blaabjerg, J. K. Pederson, E. Ritchie, and P. Nielsen, "Determination of mechanical resosnaces in induction motors by random modulation and acoustic measurement," IEEE Trans. Ind. Applicat., vol. 31, pp. 823-829, July/Aug. 1995.

[6] S. Ueda, K. Honda, T. Ikimi, M. Hombu, and A. Ueda, "Magnetic noise reduction technique for an AC motor driven by a PWM inverter," IEEE Trans. Power Electron., vol. 6, pp. 470-475, July 1991.

[7] D. A. Grant, "PWM AC motor drive employing untrasonic carrier," in Proc. IEE Conf. Power Electric Variable Speed Drives, vol. 234, 1984, pp. 237-240.

[8] M. Hombu, A. Ueda, and Y. Matsuda, "A new source GTO inverter with sinusoidal output voltage and current," IEEE Trans. Ind. Applicat., vol. IA-21, pp. 1192-1198, Sept./Oct. 1985.

[9] R. Hupe and R. Kennel, "Increase of power and reduction of noise and current ripple of inverter fed induction machines," in Proc. EPE'89, Aachen, Germany, 1989, pp. 47-50.

[10] Y. X. Chen and Z. Q. Zhu, Analysis and Control of Noise from Electrical Machines. Hangzhou, China: Zhejiang Univ. Press, 1987.

[11] Z. Q. Zhu and D. Howe, "Effect of end-shields and rotor on the natural frequencies and modes of stator of an electrical machine," in Proc. 4th Int. Conf. Electrical Machines and Drives, 1989, pp. 232-236.

[12] R. Bowe, "Advanced regular-sampled PWM control techniques for drives \& static power converters," IEEE Trans. Ind. Electron., vol. 42, pp. 367-373, Aug. 1995.

[13] H. Van Der Broeck, H. C. Skudelny, and G. V. Stanke, "Analysis and realization of a pulsewidth modulator based on voltage space vector," IEEE Trans. Ind. Applicat., vol. 24, pp. 142-149, Jan./Feb. 1988. 




W. C. Lo (M'83) received the B.Sc.(Eng.), M.Phil., and Ph.D. degrees from the University of Hong Kong, Hong Kong, in 1983, 1986 and 1996, respectively, all in electrical and electronic engineering.

Since 1995, he has lectured in the Department of Electrical and Electronic Engineering, University of Hong Kong. He previously held a number of academic posts in other universities in Hong Kong, and spent a period in industry with Telstra, Ltd., Australia. His current research interests include drives and traction, electric vehicles, electrical installations in buildings, and renewable energy.

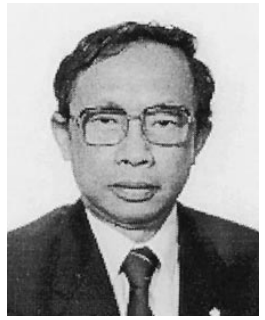

C. C. Chan (M'77-SM'77-F'92) started his professional electrical engineering career in 1959. $\mathrm{He}$ worked 11 years in industry and 26 years in academic institutions. He is currently the Head of the Department of Electrical and Electronic Engineering, University of Hong Kong, Hong Kong, the Honda Chair Professor of Engineering of the Department, and the Director of the International Research Centre for Electric Vehicles. He has been a Visiting Professor at several universities around the world. His current research interests include electrical machines, advanced electric drives, electric vehicles, power systems, and power electronic applications.

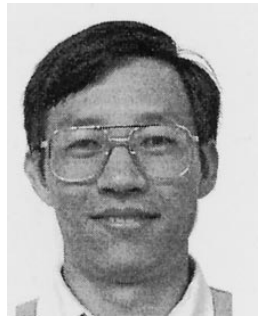

Z. Q. Zhu (M'90) received the B.Eng. and M.Sc. degrees from Zhejiang University, Hangzhou, China, and the Ph.D. degree from the University of Sheffield, Sheffield, U.K., in 1982, 1984, and 1991, respectively, all in electrical and electronic engineering.

From 1984 to 1988, he lectured in the Department of Electrical Engineering, Zhejiang University. Since 1988, he has been with the University of Sheffield, where he is currently a Senior Research Scientist in the Department of Electronic and Electrical Engineering. His current research interests include the applications, control, and design of permanent-magnet machines and drives. He has authored more than 130 published journal and conference papers.

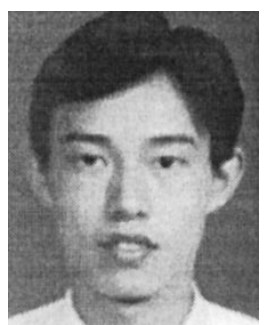

Lie Xu (M'90) received the B.Eng. degree from Zhejiang University, Hangzhou, China, and the Ph.D. degree from the University of Sheffield, Sheffield, U.K., in 1993 and 1999, respectively, both in electrical engineering.

Since 1999, he has been a Research Fellow in the Inter-University Glasgow-Strathclyde Centre for Economic Renewable Power Delivery, Glasgow, U.K. His research interests include power electronics, motor drives, and FACTS devices.



David Howe received the B.Tech and M.Sc. degrees from the University of Bradford, Bradford, U.K., and the Ph.D. degree from the University of Southampton, Southampton, U.K., in 1966, 1967, and 1974, respectively, all in electrical power engineering.

$\mathrm{He}$ has held academic posts at Brunel and Southampton Universities, and spent a period in industry with NEI Parsons Ltd., working on electromagnetic problems related to turbogenerators. $\mathrm{He}$ is currently Lucas Varity Professor of Electrical Engineering at the University of Sheffield, where he heads the Electrical Machines and Drives Research Group. His research activities span all facets of controlled electrical drive systems, with particular emphasis on permanent-magnet excited machines. He is the author of more than 200 publications in the fields of machines, drives, and motion control systems.

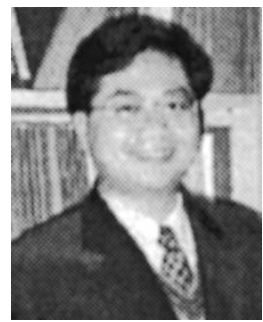

K. T. Chau (M'89) received the B.Sc.(Eng.), M.Phil., and Ph.D. degrees from the University of Hong Kong, Hong Kong, in 1983, 1986 and 1996, respectively, all in electrical and electronic engineering.

Since 1995, he has lectured in the Department of Electrical and Electronic Engineering, University of Hong Kong. He has been a Reviewer for three international journals, as well as serving as chairs and organizing committee members for various international conferences in the areas of electric vehicles, electric drives, industrial electronics, and mechatronies. 